\title{
Consideration of contribution of hot-electron injection to the resistive switching of sputter-deposited silicon oxide film
}

\author{
Yasuhisa Omura \\ ORDIST, Kansai University, Osaka, Japan
}

\begin{tabular}{l} 
Article Info \\
\hline Article history: \\
Received Jun 9, 2021 \\
Revised Oct 14,2021 \\
Accepted Oct 27, 2021 \\
\hline
\end{tabular}

\section{Keywords:}

Bipolar switching

Hafnium oxide

Hot electron

Resistive switching

Silicon oxide

Sputter-deposited

Unipolar switching

\begin{abstract}
This paper considers the contribution of hot electrons to the resistive switching of sputter-deposited silicon oxide films based on experiments together with semi-2D Monte Carlo simulations. Using various device stack structures, this paper examines the impact of hot-electron injection on resistive switching, where conduction-band offset and fermi-level difference are utilized. Support is found for the predictions that hot-electron injection reduces the switching voltage and this should reduce the dissipation energy of switching. It is predicted that two-layer metal stacks can significantly reduce the number of oxygen vacancies in the sputter-deposited silicon oxide film after the reset process. It is also demonstrated that, in unipolar switching, the number of E' or E" centers of the sputter-deposited silicon oxide film is relatively large.
\end{abstract}

This is an open access article under the CC BY-SA license.

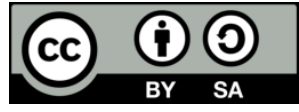

\section{Corresponding Author:}

Yasuhisa Omura

ORDIST, Kansai University

3-3-35 Yamate-cho, Suita, Osaka, Japan

Email: omuray@kansai-u.ac.jp

\section{INTRODUCTION}

Many recent papers have demonstrated and discussed in detail the resistive switching mechanisms of sputter-deposited silicon oxide films [1]-[16] because this structure dispenses with the rear metal and the transition metal. Most of those papers addressed the role of silicon sub-oxide $\left(\mathrm{SiO}_{\mathrm{x}}\right)$ [1]-[4], [6], [7], [9], [10] because it is anticipated that the unstable chemical states of non-stoichiometric silicon oxide can create conductive paths [17], that can be recovered, inside the film. However, it is not yet clear how the silicon suboxide region can trigger resistive switching, and whether the silicon sub-oxide region is the only determiner of resistive switching [12], [13]. The author's previous paper demonstrated that hot-electron injection from the Si substrate had the potential to trigger resistive switching from sputter-deposited Si oxide films [18]; the stack of $\beta-\mathrm{FeSi}_{2} / \mathrm{Si}$ substrate with the conduction-band energy offset of $0.2 \mathrm{eV}$ was used as the bottom electrode [19]. However, it did not definitively elucidate why unipolar switching is infrequent in sputterdeposited silicon oxide films [20], [21], even though it is not a transition-metal oxide.

Since observations of unipolar switching in silicon oxide films have been reported in recent studies [14], [22], the author discussed what might happen in unipolar switching in [21], [22] by analyzing the transient processes present in resistive switching. However, the primary reason why unipolar switching is not a stable process in sputter-deposited silicon oxide films remains unclear. Therefore, while potential switching mechanisms have been investigated from the aspect of chemistry, resistive switching in silicon oxide films may still be governed by a couple of unknown, but important, phenomena [23]-[26]. Therefore, it is expected that further studies on such resistive switching phenomena are needed to attain a more substantial 
understanding of not only degradation mechanisms [27] but also recovery mechanisms of silicon oxide films and related materials [23]-[26].

This paper carries out semi-two-dimensional (S2D) Monte Carlo simulations on the resistive switching of sputter-deposited silicon oxide films [21], [22], [28], [29] in order to elucidate the importance of carrier energy in switching. Although Monte-Carlo-based studies have already been performed to analyze the switching behavior of various metal oxide films [21], [30]-[32] with verification by experiments [18]-[20], [26], few have targeted sputter-deposited silicon oxide films [21], [23] and the impact of hot-electron injection on resistive switching has not been discussed so far. This paper focuses on how the initial carrier energy influences the resistive switching behavior of silicon oxide films.

\section{DEVICE STRUCTURE SIMULATED}

This study reports the results of Monte Carlo simulations of various device structures, some of which were used in the author's prior experiments [18]-[20]. The device structures assumed in this paper are shown in Figure 1 [18]-[20], where Figure 1(a) shows the device having a stack of Au/silicon oxide/n-Si substrate (called device A, control device), Figure 1(b) shows the device having a stack of Au/silicon oxide/n-type $\beta$-FeSi $2 / n-S_{i}$ substrate (called device B), Figure 1(c) shows Hf/Au/silicon oxide/n-Si substrate (called device C), and Figure 1(d) shows $\mathrm{Hf} / \mathrm{Au} /$ silicon oxide/n-type $\beta-\mathrm{FeSi}_{2} / \mathrm{n}-\mathrm{Si}$ substrate (called device D). Devices $\mathrm{B}, \mathrm{C}$, and D are considered here in order to illuminate the impact of the high-energy fraction of electrons injected from the electrode. Device parameters and physical parameters assumed are summarized in Table 1 , where $t$ thicknesses of $\beta-F_{e S i_{2}}$ and $\mathrm{Au}$ films in devices B, C, and D are derived from the mean free path of electrons.

Device $\mathrm{A}$ is the control device used in the experiment to demonstrate that a simple bias condition fails to yield reliable unipolar switching [18], [20]. Device B is the test structure used in the experiment to demonstrate that unipolar switching and bipolar switching can be easily realized [19]. Device $C$ is another test structure assumed here to demonstrate that the hot-electron injection supports resistive switching. Device $\mathrm{D}$ merges the architectures of device $\mathrm{B}$ and device $\mathrm{C}$ to examine the potential of hot electron injection in lowering the supply voltage.

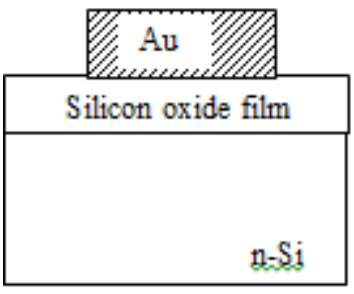

(a)

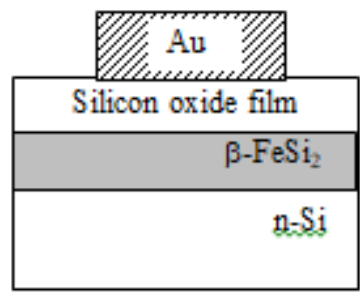

(b)

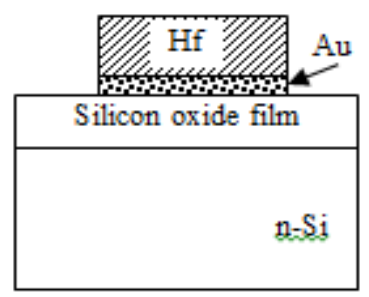

(c)

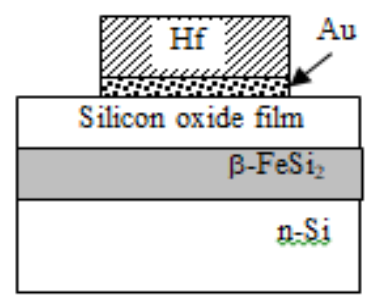

(d)

Figure 1. Fundamental device structures used in simulations: (a) device A: Au/Si oxide/n-Si, (b) device B: $\mathrm{Au} / \mathrm{Si}$ oxide/ $\beta-\mathrm{FeSi}_{2} / \mathrm{n}-\mathrm{Si}$, (c) device C: $\mathrm{Hf} / \mathrm{Au} / \mathrm{Si}$ oxide/n-Si, and (d) device D: Hf/Au/Si oxide/ $\beta-\mathrm{FeSi}_{2} / \mathrm{n}-\mathrm{Si}$

Table 1. Device parameters used in experiments and physical parameters for Monte Carlo simulations, silicon oxide film is assumed as the insulator film

\begin{tabular}{ll}
\hline Parameters & Values [units] \\
\hline Silicon oxide thickness & $8.0[\mathrm{~nm}]$ \\
$\mathrm{Si}-\mathrm{O}$ bond length & $0.155[\mathrm{~nm}][33]$ \\
Effective mass of electrons & $0.3 \mathrm{~m}_{0}$ \\
Dielectric constant $\left(\mathrm{SiO}_{2}\right)$ & $3.8 \varepsilon_{\mathrm{o}}[\mathrm{F} / \mathrm{m}]$ \\
$\beta-F e S i_{2}$ thickness & $20[\mathrm{~nm}]$ \\
Conduction band offset & $0.2[\mathrm{eV}]$ \\
$\quad$ between $\beta-F e S i_{2}$ and $\mathrm{Si}$ & \\
Conduction band offset & $3.1[\mathrm{eV}]$ \\
$\quad$ between $\mathrm{SiO}_{2}$ and $\mathrm{Si}$ & $10[\mathrm{~nm}]$ or 300 [nm] \\
Au layer thickness & $5.4[\mathrm{eV}][34]$ \\
Work function of Au film & $3.9[\mathrm{eV}][35]$ \\
Work function of Hf film & $-5.0 \mathrm{to}+6.0[\mathrm{~V}]$ \\
Stress voltage $(\mathrm{V})$ & $5.7[\mathrm{eV}][36]$ \\
Degradation energy $\quad\left(\mathrm{SiO}_{2}\right)$ & $6.5[\mathrm{eV}][37]$ \\
Ov creation energy $\quad\left(\mathrm{SiO}_{2}\right)$ & $1.3[\mathrm{eV}][38]$ \\
E' or E' creation energy $\left(\mathrm{SiO}_{2}\right)$ & $1.7[\mathrm{eV}][38]$ \\
Ov annihilation energy $\left(\mathrm{SiO}_{2}\right)$ & \\
\hline$\varepsilon_{\mathrm{o}}$ is the dielectric constant of vacuum $)$
\end{tabular}




\section{MONTE CARLO SIMULATIONS AND PHYSCAL MODELS}

\subsection{Fundamental assumptions for Monte Carlo simulations}

Our previous papers [18]-[20] suggested that hot electrons injected from the Si substrate significantly contributed to the stable resistive switching of sputter-deposited silicon oxide films. Accordingly, this paper details how important the injection of high-energy electrons is in establishing resistive switching. For simulating resistive switching, this paper assumes that stress-induced degradation of the silicon oxide film induces resistive switching; that is, constant voltage stress is assumed.

Figure 2(a) shows a schematic of the device structure assumed here (control device A) and Figure 2(b) shows the simplified lattice image of silicon and oxygen atoms in the silicon oxide film. In Figure 2(a), electrons are injected from the top metal electrode or the Si substrate. Though the crystalline image shown in Figure 2(b) is not strictly appropriate for amorphous silicon oxide film, it is a nominal assumption to permit the simulations.

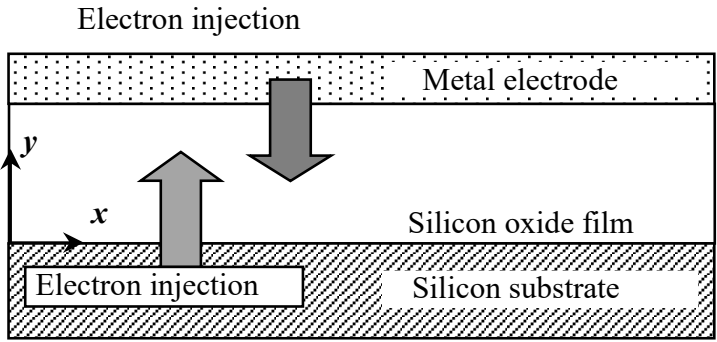

(a)

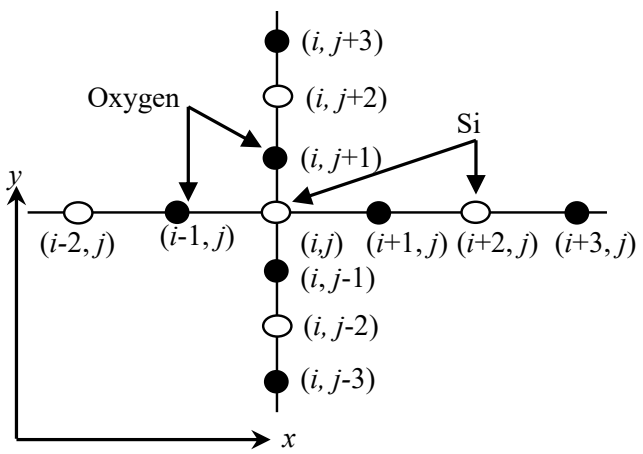

(b)

Figure 2. Device structure assumed and chemical bonding image of oxide material. (a) Device structure (device A) and (b) Chemical bonding image of oxide material. Brackets reveal the lattice sites considered in the simulations

Though this simplified assumption is not physically sufficient, it was already applied to the past simulation to explain aspects of the stress-induced-leakage current (SILC) of sub-5-nm-thick $\mathrm{SiO}_{2}$ films [39]. Simulation results successfully elucidated some mechanisms in addition to the past understanding, and they reproduced quantitatively I-V characteristics of degraded $\mathrm{SiO}_{2}$ films. Therefore, this paper applied this simulation technique to the following simulations. Here it is assumed that the silicon oxide is $8.0 \mathrm{~nm}$ thick. The physical thickness was chosen mainly due to the long-term reliability history of silicon oxide films [40].

In this paper, it is assumed that the sputter-deposited Si oxide film has intrinsic defects (the initial

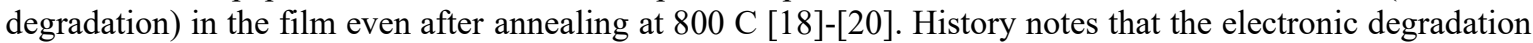
of silicon oxide film is often characterized by a change in Si-O bond angle and bond distance [33]. When the Si-O bond site receives the degradation energy of $5.7 \mathrm{eV} \mathrm{[36]} \mathrm{by} \mathrm{collisions} \mathrm{of} \mathrm{carriers} \mathrm{or} \mathrm{external} \mathrm{high-energy}$ particles, it is considered that it reaches the worst case of the bond state without bond breakage. Since most chemical bond states of sputter-deposited silicon oxide films are not ideal, it is anticipated that each bond site is somewhat degraded; that is, the site has initially exhibits some part of the energy-based degradation. Thus, Sections 4.1 and 4.2 tentatively assume that the initial level of degradation energy of the sputter-deposited silicon oxide film increases linearly from $2.8 \mathrm{eV}$ (at the bottom interface) to $3.1 \mathrm{eV}$ (at the top surface). This assumption is acceptable because the top surface of sputter-deposited film is more frequently degraded in comparison to the bottom.

\subsection{Physical models for the resistive switching in simulations}

Physical images of the electroforming process and reset process are illustrated in Figures 3 and 4, respectively. Carriers are injected from the top electrode or the Si substrate. Since carrier movement is limited to a single atomic layer slice for the purpose of simulation simplicity, the calculations of carrier traveling and inelastic scattering are carried out at every atomic site of the slice.

For silicon oxide films, the present author's model successfully explains unipolar switching without the direct Joule effect [21] because the energy to change the electronic state of atomic site ranges from 1 to 6 eV [19], [21]. Generally speaking, hot electrons have higher potential than "cold" electrons, so they can provide the energy to atomic sites for resistive switching. It is considered that the simple Joule effect is not 
the primary mechanism for the resistive switching of sputter-deposited silicon oxide films, though the conventional Joule effect may be a secondary mechanism.

When an electron loses a part of its energy at an atomic site, the energy accumulates in the bond state the atom possesses. When the accumulated energy reaches a certain value, the bond is broken as is mentioned in the previous sub-section. As mentioned above, it is assumed for simplicity that resistive switching does not alter significantly the local lattice temperature, and that most energy dissipated by electrons is consumed in chemical reactions. In other words, since the simulation does not take account of diffusion of chemicals [23]-[27], such as $\mathrm{H}^{+}, \mathrm{OH}^{-}$, and $\mathrm{H}_{2} \mathrm{O}$, details of the chemical reactions are not discussed.

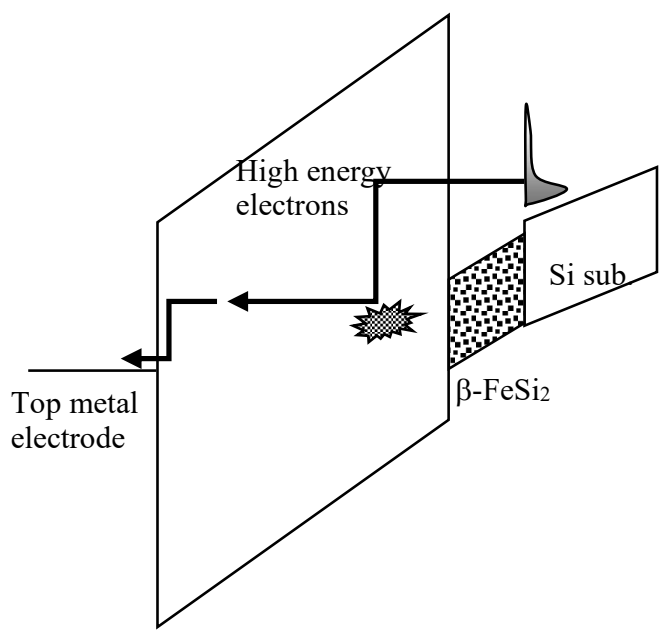

(a)



(b)

Figure 3. Physical images of the electroforming process and the reset process of the film. (a) Device B is assumed. A positive voltage is applied to the top electrode. Hot electrons are injected from the Si substrate and (b) Device $\mathrm{C}$ is assumed. A negative voltage is applied to the top electrode and hot electrons are injected from the top metal electrode

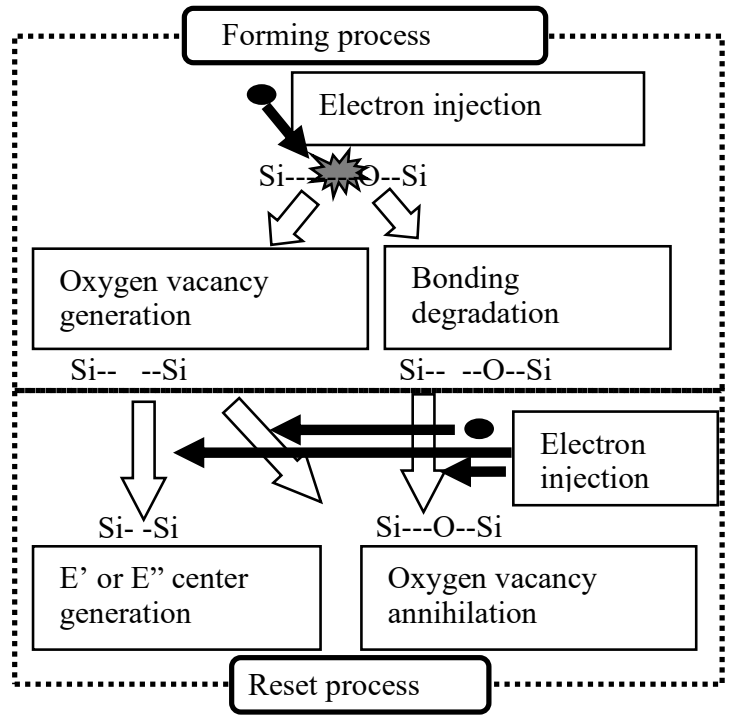

Figure 4. Physical images of electroforming process and reset process inside the silicon oxide films. For the hafnium oxide, a similar physical image is assumed except for E' or E" centers

To take account of the initial energy distribution of electrons of the Si substrate and the metal electrode we adopt the Fermi-Dirac function (See Figures 3(a) and 3(b)). In simulations for device B, the 
conduction band offset between the $\beta-\mathrm{FeSi}_{2}$ and the $\mathrm{Si}$ substrate is considered in electron injection from the Si substrate (See Figure 3(a)), and in simulations for device C, the Fermi level difference of $\mathrm{Hf}$ and $\mathrm{Au}$ is considered (See Figure 3(b)) because it is expected that most electrons are injected from the top electrode (Hf). Device D is examined in order to investigate whether merging devices B and C provides any benefit.

Figure 4 summarizes the phenomena assumed here. As shown in Figure 4, in the reset process, it is expected that some of the oxygen vacancies recover the Si-O-Si bond state, otherwise they change to E' or E" centers in the silicon oxide film [19], [36]. In the simulations of the reset process, the generation of E' or E" centers (only for silicon oxide) and the annihilation of oxygen vacancies are counted.

In the following simulations, it is assumed that oxygen vacancies created by collisions of carriers at the atomic site produce seeds that form the conductive filament, and that the critical degradation fraction of atomic sites is about $30 \%$ [41]. Therefore, the number of carriers injected is controlled so that the final degradation fraction of atomic sites is over $30 \%$, but less than $50 \%$. In the simulations, it assumed that the degradation can be represented by the total energy accumulated by inelastic collisions of electrons. The energy at the atomic site is integrated, and the number of atomic sites whose energy is higher than the bond-breakage energy is counted. When the degradation fraction is larger than 0.3 , the simulation is stopped. Since it is expected that a voltage pulse for writing the data is applied to the device in practical circuits, the number of electrons injected during the pulse interval is calculated and the degradation fraction is counted after application of the voltage pulse.

We have already demonstrated XPS analysis results of excess silicon inclusions in a fabricated sputter-deposited silicon oxide film [18]-[20]. It was suggested that excess Si precipitates enabled resistive switching because sputter-deposited silicon oxide films without the Si precipitates didn't show stable resistive switching [20]. Since it is expected that large amounts of Si precipitates help the local breakdown or degradation and finally the creation of the conductive filament, this assumption is reasonable [21]. In the simulations, the number of electrons injected from the electrode and their energy-dependent distribution are taken into account because it is anticipated that those parameters strongly influence the switching behavior. Those parameters alter the initial tunnel current through the oxide film.

The effective electron current density $(J)$ is estimated as:

$$
J \propto f_{b}\left(V_{o x}\right) \int_{E_{c}}^{E_{m}} D\left(E-E_{c}\right) f_{F D}(E) T_{\text {insulator }}(E) d E
$$

where $D(E)$ is the effective density of states of the Si substrate or metal electrode $(\mathrm{Au}), f_{\mathrm{FD}}(E)$ is the FermiDirac function, $T_{\text {insulator }}(E)$ is the tunnel probability, and $f_{b}\left(V_{o x}\right)$ is the bias factor given by the voltage drop $\left(V_{o x}\right)$ across the insulator film. In carrier injection from the Si substrate and the top electrode, the effective energy barrier of insulator film is considered as shown in (1); that is, the tunneling probability in the direct tunneling condition and the Fowler-Nordheim tunneling condition are taken into account. In addition, the bias factor $f_{b}\left(V_{o x}\right)$ alters the number of carriers injected from the electrode. Total number of carriers injected depends on applied voltage and ranges from $10^{4}$ to $10^{5}$ per atomic slice.

\section{RESULTS AND DISCUSSIONS}

\subsection{Unipolar switching potential of sputter-deposited si oxide films}

This subsection starts with device A. In order to examine the unipolar switching process, in the electroforming process, a positive voltage $(4.0 \mathrm{~V})$ is applied to the top electrode, and in the reset process, a positive voltage $(4.0 \mathrm{~V})$ is again applied to the top electrode. Simulation results are shown in Figure 5. Figure 5(a) shows the simulation result of the electroforming process, where electrons are injected from the $\mathrm{Si}$ substrate. The degradation fraction of atomic sites is $35.2 \%$. A clear conductive filament (bold white line) is generated. Figure 5(b) shows the result of the reset process, where electrons are injected from the Si substrate. Though the internal state of degradation is almost unchanged, the simulation result reveals that 8 E' or E" centers $(\mathrm{O}-\mathrm{Si}=\mathrm{Si}-\mathrm{O})$ are created and 79 oxygen vacancies are annihilated in the reset process. Since these values are statistically determined, they vary slightly in each simulation. However, their overall low values are meaningful.

The result suggests that combining this stack structure and the bias configuration is not successful in achieving unipolar switching, as was clearly demonstrated in our previous experiments [20]. Since it is anticipated that the high-energy fraction of electrons injected from the Si substrate heavily damage the film, it is reasonable to expect that the reset process will be unable to recover the degradation generated in electroforming; this is supported by the paucity of E' or E" centers generated and the very small number of oxygen-vacancy annihilations. This is the most obvious reason why unipolar switching does not occur readily under positive voltage stress.

Next, the impact of hot-electron injection is examined by simulations of device B. In order to examine the potential of unipolar switching process, three-different simulations were carried out. The most interesting result is shown in Figure 6, where, for the electroforming process, a positive voltage $(3.5 \mathrm{~V})$ is applied to the top electrode, while for the reset process a positive voltage $(4.0 \mathrm{~V})$ is applied again to the top electrode. 
Figure 6(a) shows the simulation result of the electroforming process, where hot electrons are injected from the Si substrate. In this configuration, the excess energy of electrons injected from the Si substrate is about $0.3 \mathrm{eV}$. The degradation fraction of atomic sites is $34.5 \%$. A clear conductive filament (bold white line) is generated. Figure 6(b) shows that yielded by the reset process, where hot electrons are injected from the Si substrate. The degradation fraction of atomic sites is 3.5\%. The important point in Figure 6(a) is the fact that the degradation fraction in the post-electroforming state is roughly the same as that in Figure 5(a) in spite of the lower stress voltage. This clearly demonstrates that the high-energy fraction of electrons injected from the $\mathrm{Si}$ substrate plays an important role in the reset process. It is worthy to note that the number of E' or E" centers (O$\mathrm{Si}=\mathrm{Si}-\mathrm{O})$ created is 1353 and the number of annihilated oxygen vacancies is 3700 in Figure $6(\mathrm{~b})$. This reveals that most of the excess energy of electrons injected is consumed in the annihilation of oxygen vacancies; that is, the impact of the excess energy of electrons is significant even if its value is at most $0.3 \mathrm{eV}$.

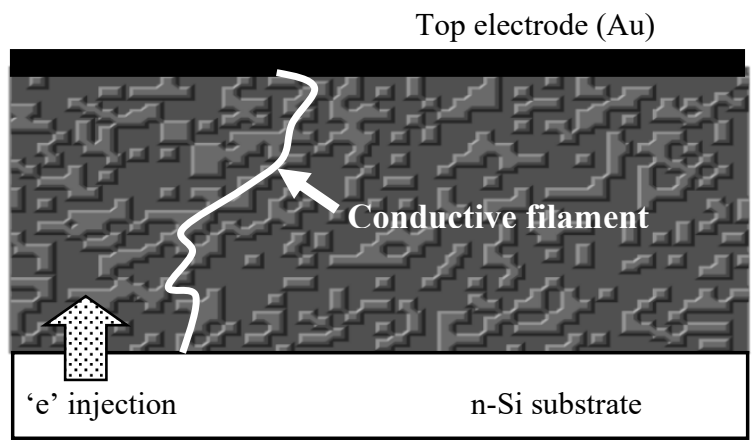

(a)

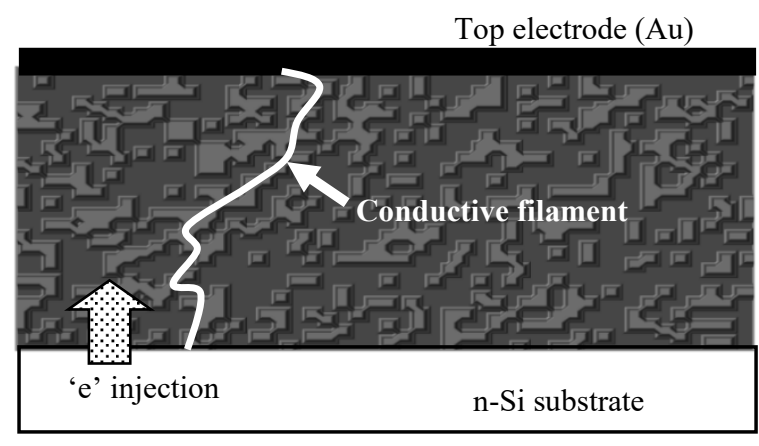

(b)

Figure 5. Unipolar-switching simulation results. Device A is assumed. Electrons are injected from the Si substrate. Degradation images of 8-nm-thick silicon oxide film are shown. Carrier injection is indicated by arrows. (a) After the 1 st stress and (b) After the $2^{\text {nd }}$ stress

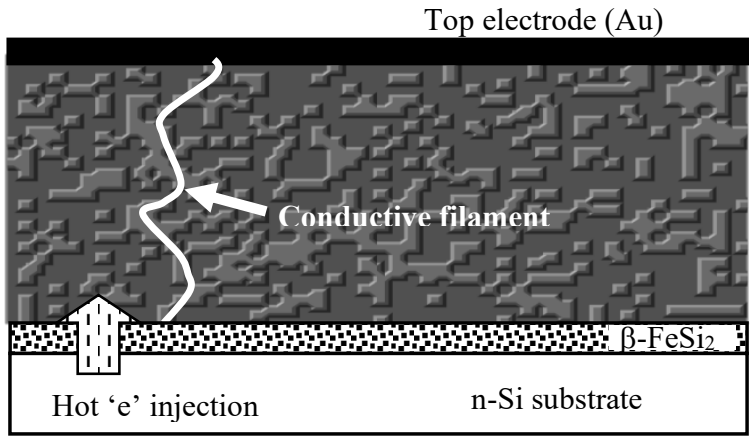

(a)

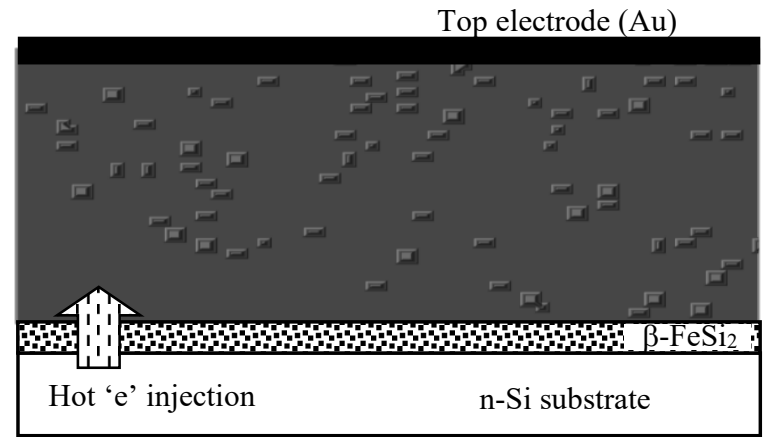

(b)

Figure 6. Unipolar-switching simulation results. Device B is assumed. Hot electrons are injected from the Si substrate. Degradation images of 8-nm-thick silicon oxide film is shown. Carrier injection is revealed by arrows. (a) Post-electroforming state and (b) Post-reset state

Next, the other case of unipolar switching is considered. Device A is taken as the control device. A negative voltage of $-4.5 \mathrm{~V}$ is applied to the top metal electrode in the electroforming process, and a negative voltage of $-4.5 \mathrm{~V}$ is applied to the top metal electrode in the reset process. Simulation results are shown in Figure 7. Figure 7(a) shows the simulation result of the electroforming process, where electrons are injected from the top metal electrode $(\mathrm{Au})$. The degradation fraction of atomic sites is $41.5 \%$. A clear conductive filament (bold white line) is generated. Figure 7(b) shows that of the reset process, where electrons are also injected from the top electrode $(\mathrm{Au})$. Though the internal situation of degradation is almost unchanged, the simulation results reveals that 84 E' or E" centers $(\mathrm{O}-\mathrm{Si}=\mathrm{Si}-\mathrm{O})$ are created and 313 oxygen vacancies are annihilated in the reset process. This is very similar to the result in Figure 5(b). 
The result confirms our previous finding that suggests that this combination of stack structure and bias configuration is not practical for realizing unipolar switching [42]. It is anticipated that unipolar switching fails for basically the same reason as given for Figure 5. Using the same approach, we examine the impact of hot-electron injection by simulating device C. An interesting result is shown in Figure 8, where, in the electroforming process, a negative voltage $(-3.65 \mathrm{~V})$ is applied to the top electrode, and a negative voltage $(-4.3 \mathrm{~V})$ is applied to the top electrode in the reset process.

Figure 8(a) shows the simulation result of the electroforming process, where hot electrons are injected from the top metal electrode (Hf). The degradation fraction of atomic sites is $36.8 \%$. A clear conductive filament (bold white line) is generated. Figure 8(b) shows the result of the reset process, where hot electrons are injected from the top metal electrode (Hf). The degradation fraction of atomic sites is $3.0 \%$. The important point in Figure 8(a) is the large degradation fraction in the post-electroforming state (roughly the same as that in Figure 7(a)) in spite of the lower stress voltage. It is considered that the large degradation fraction in Figure 8(a) is due to the large difference in Fermi levels of Hf and Au (See Table 1); the excess energy of electrons injected from the top metal electrode (Hf) is about $1.5 \mathrm{eV}$. We can consider that the highenergy electrons injected from the top metal electrode play an important role in the reset process. In the simulation, the number of E' or E" centers created is 1623 and the number of annihilated oxygen vacancies is 3598 in Figure 8(b). It is also worthy to note that there are fewer E' or E" centers than annihilated oxygen vacancies; that is, a large part of the excess energy of electrons is consumed in the annihilation of oxygen vacancies.

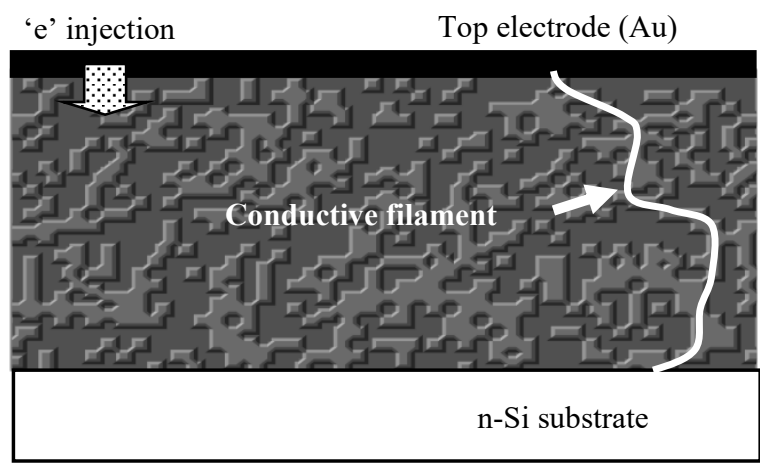

(a)

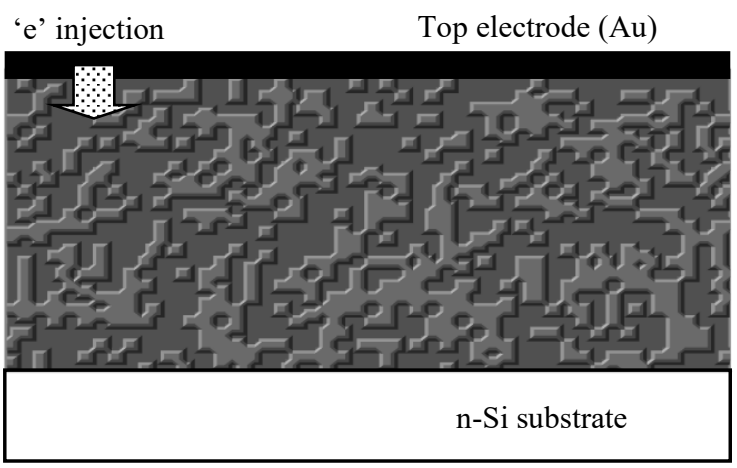

(b)

Figure 7. Unipolar-switching simulation results. Device A is assumed. Electrons are injected from the top metal electrode $(\mathrm{Au})$. Degradation images of 8-nm-thick silicon oxide film is shown. Carrier injection is revealed by arrows. (a) After the $1^{\text {st }}$ stress and (b) After the $2^{\text {nd }}$ stress

Hot 'e' injection

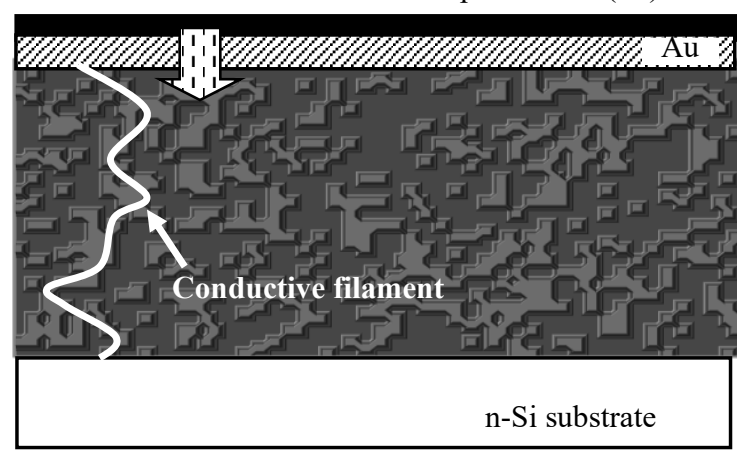

(a)

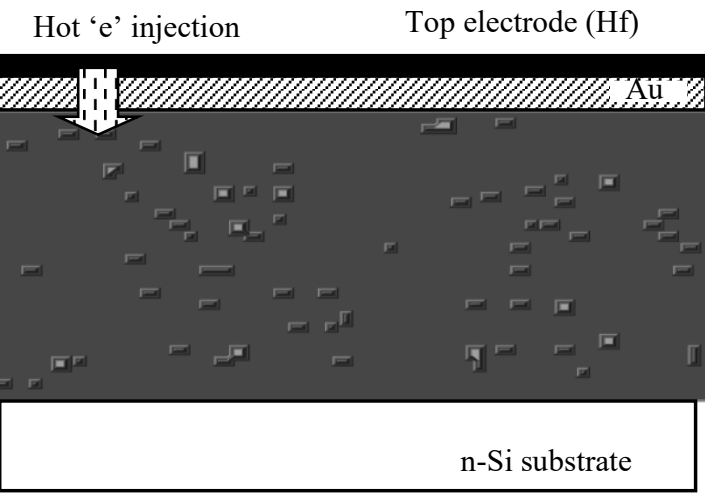

(b)

Figure 8. Unipolar-switching simulation results. Device $\mathrm{C}$ is assumed. Hot electrons are injected from the top metal electrode (Hf). Degradation images of 8-nm-thick silicon oxide film is shown. Carrier injections are revealed by arrows. (a) Post-electroforming state and (b) Post-reset state 


\subsection{Bipolar switching potential of sputter-deposited si oxide films}

Some aspects of bipolar switching in sputter-deposited silicon oxide films are discussed with reference to the previous sub-section. At first, device A is considered. In order to examine the bipolar switching process, the electroforming process applies a negative voltage $(-4.0 \mathrm{~V})$ to the top electrode, while the reset process applies a positive voltage $(4.0 \mathrm{~V})$ to the top electrode. Simulation results are shown in Figure 9.

Figure 9(a) shows the simulation result of the electroforming process, where electrons are injected from the top metal electrode. The degradation fraction of atomic sites is $38.2 \%$. A clear conductive filament (bold white line) is generated. Figure 9(b) shows the result of the reset process, where electrons are injected from the Si substrate. Though the internal degradation result is basically unchanged, the simulation results reveal that 14 E' or E" centers are created and 84 oxygen vacancies are annihilated in the reset process.



(a)



(b)

Figure 9. Bipolar-switching simulation results. Device A is assumed. Degradation images of 8-nm-thick silicon oxide film are shown. Electrons are injected from the trop electrode for the electroforming process, and they are injected from the n-Si substrate for the reset process. (a) After the $1^{\text {st }}$ stress and (b) After the $2^{\text {nd }}$ stress

The above result suggests that this stack structure and bias configuration is ineffective in terms of bipolar switching. Other simulation results (not shown here) reveal that a much higher voltage is needed in the reset process to achieve successful switching, which was clearly seen in our previous experiments [42]. In this case, it has been found that a relatively large number of electrons are injected from the top metal electrode in the electroforming process, making a higher voltage $(\sim 6 \mathrm{~V})$ essential if the reset process is to realize adequate switching performance. This is supported by the paucity of E'- or E'-centers generated and very few oxygen-vacancy annihilation events in the reset process at $4 \mathrm{~V}$.

To further address this point, device $\mathrm{B}$ is examined. The electroforming process applies a negative voltage $(-4.0 \mathrm{~V})$ to the top electrode, while the reset process applies a positive voltage $(4.0 \mathrm{~V})$ to the top electrode. Simulation results are shown in Figure 10. Figure 10(a) shows the simulation result of the electroforming process, where electrons are injected from the top metal electrode. The degradation fraction of atomic sites is $34.2 \%$. A clear conductive filament (bold white line) is generated. Figure 10(b) shows the results of the reset process, wherein hot electrons are injected from the $\mathrm{Si}$ substrate. The conductive filament has disappeared. The degradation fraction of atomic sites is 3.6\%. The simulation results reveal that 1345 E' or E" centers are created, with 3677 annihilated oxygen vacancies. The above result reveals that this combination of stack structure and bias configuration can realize stable bipolar switching, and that the contribution of hot electrons to the successful switching realization is significant. A comparison to the case shown in Figure 9, indicates that the use of hot electrons is very meaningful in terms of lowering the switching voltage.

To discuss this in more detail, device $\mathrm{C}$ is examined. The electroforming process applies a negative voltage $(-3.65 \mathrm{~V})$ to the top electrode, while the reset process applies a positive voltage $(3.7 \mathrm{~V})$ to the top electrode. Simulation results are shown in Figure 11. Figure 11(a) shows the simulation result of the electroforming process, where hot electrons are injected from the top metal electrode. The degradation fraction of atomic sites is $38.3 \%$. The degradation fraction of atomic sites is larger than that in Figure 10(a) even though the top-electrode voltage is lower than that in Figure 10(a). Although no clear conductive filament is created, sufficient degradation is obtained as shown in Figure 11(a). Figure 11(b) shows that in the reset process, electrons are injected from the $\mathrm{Si}$ substrate. The degradation fraction of atomic sites is drastically reduced (1.1\%). The simulation results reveal that the number of E' or E" centers created is 908 , and the number of annihilated oxygen vacancies is 4098. The reduction in oxygen vacancies is more significant than the creation of E' or E" centers. 


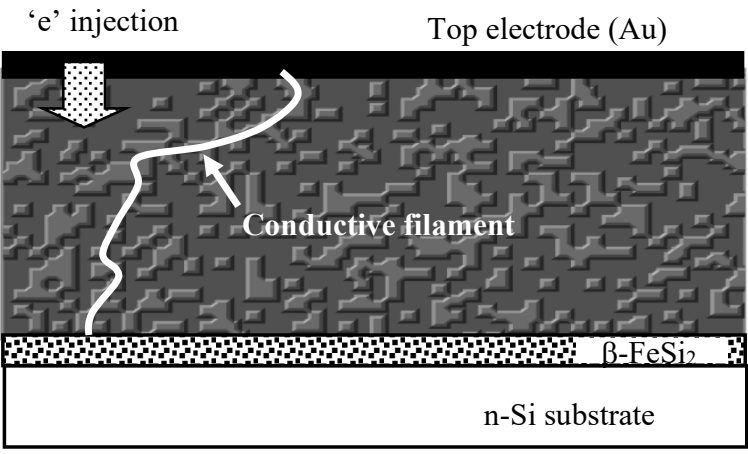

(a)

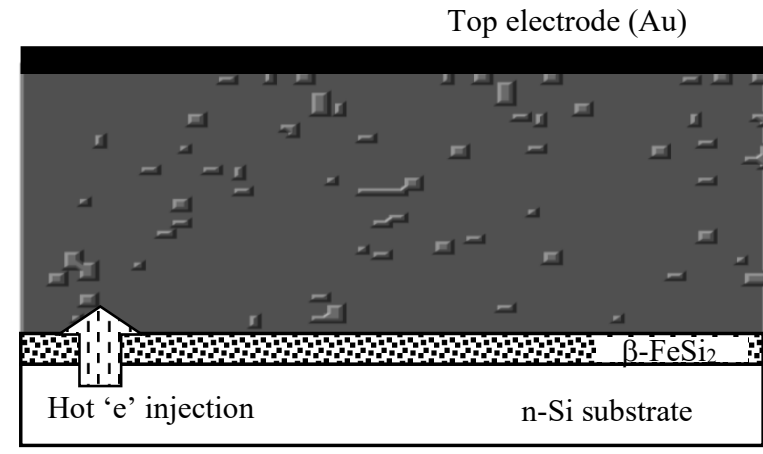

(b)

Figure 10. Bipolar-switching simulation results. Device B is assumed. Degradation images of 8-nm-thick silicon oxide film are shown. Electrons are injected from the top electrode in the electroforming process, and hot electrons are injected from the n-Si substrate in the reset process. (a) Post-electroforming state and (b) Post-reset state

Hot 'e' injection

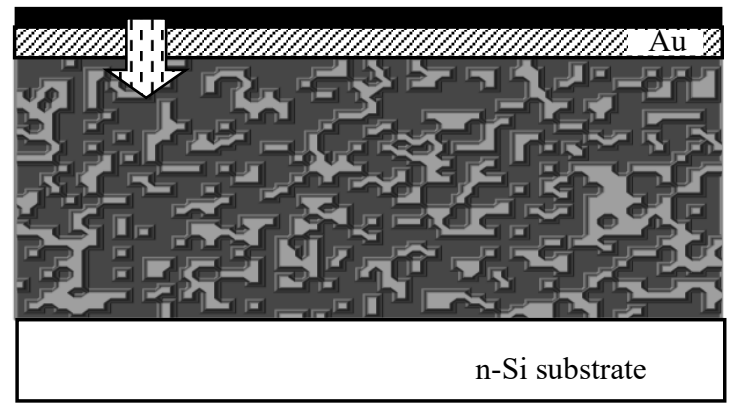

(a)

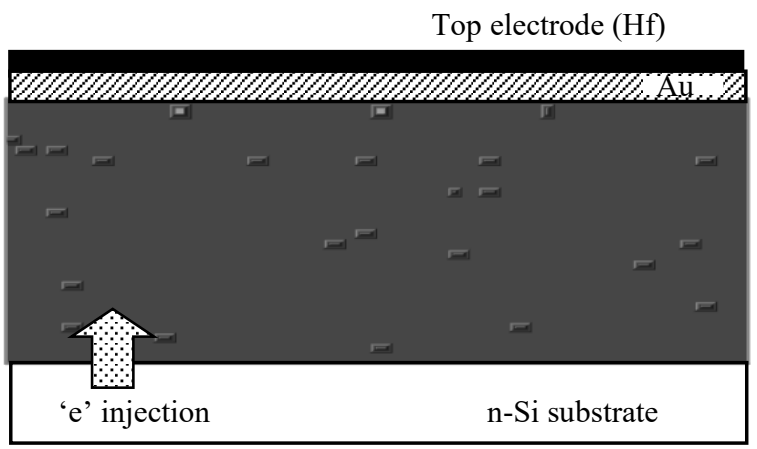

(b)

Figure 11. Bipolar-switching simulation results. Device C is assumed. Degradation images of 8-nm-thick silicon oxide film are shown. Hot electrons are injected from the top electrode in the electroforming process, and they are injected from the n-Si substrate in the reset process. (a) Post-electroforming state and (b) Post-reset state

Finally, device D, a merger of device B and device C, is examined in order to consider how dual hot-electron injection can improve the switching behavior. For the electroforming process, a negative voltage $(-3.5 \mathrm{~V})$ is applied to the top electrode, and for the reset process, a positive voltage $(3.5 \mathrm{~V})$ is applied to the top electrode. Simulation results are shown in Figure 12.

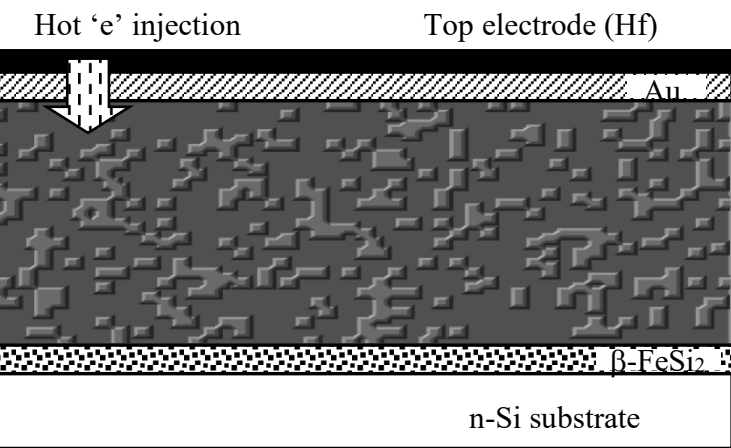

(a)

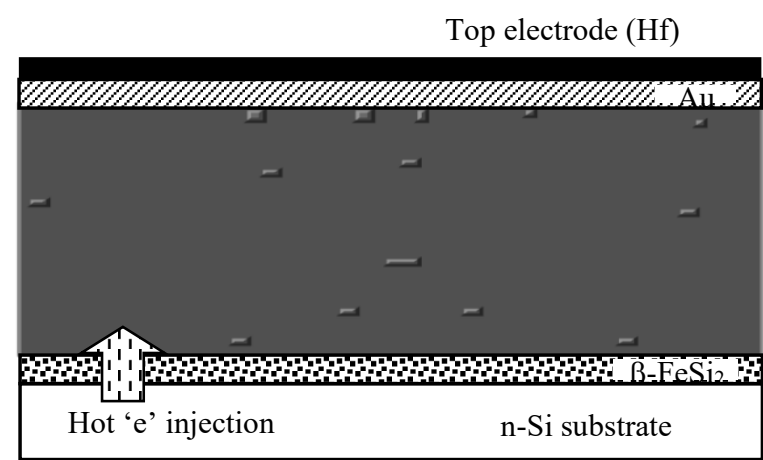

(b)

Figure 12. Bipolar-switching simulation results. Device D is assumed. Degradation images of 8-nm-thick silicon oxide film are shown. Hot electrons are injected from the top electrode for the electroforming process, and they are injected from the n-Si substrate for the reset process. (a) Post-electroforming state and

$$
\text { (b) Post-reset state }
$$


Figure 12(a) shows the simulation result of the electroforming process, where hot electrons are injected from the top metal electrode. The degradation fraction of atomic sites is $34.9 \%$. Though no clear conductive filament is generated, sufficient degradation is obtained. Figure 12(b) shows that the reset process injects hot electrons from the $\mathrm{Si}$ substrate and that the degradation in the film is almost completely eradicated. The degradation fraction of atomic sites is $0.65 \%$. The simulation results reveal that the number of E' or E" centers created is 824 , and the number of annihilated oxygen vacancies is 4174 . The results show a significant reduction in oxygen vacancies, which is an interesting point similar to that found with device C. In addition, it is worthwhile noting that the number of E' or E" centers created is much smaller than that of annihilated oxygen vacancies, which is similar to the result shown in Figure 11(b). It is suggested that the high-energy component of electrons injected from the Si substrate contributes significantly to the reduction in oxygen vacancies even for the case without hot-electron injection from the Si substrate.

\subsection{Comprehensive discussion}

All simulation results are summarized in Table 2. The following points, not discussed in the above sections, are introduced in Table 2.

a) Unipolar switching by a single silicon oxide film is not easy without the aid of hot-electron injection. This is very clear from a comparison of Figures 5 and 6, and of Figures 7 and 8.

b) Bipolar switching from a single silicon oxide film is not easy without the aid of hot-electron injection. This is very clear from a comparison of Figures 9 and 10.

c) The switching voltage of the bipolar switching of single silicon oxide film is not easily lowered, even with the aid of hot carrier injection. This is seen by comparing Figures 9, 10, 11, and 12 .

d) Devices C and D are promising for bipolar switching in future memory applications because the number of E' or E" centres in the post-reset process is smaller than that of device B; decreasing the number, lowers the leakage current.

Table 2. Summary of simulation results (U: unipolar, B: bipolar)

\begin{tabular}{|c|c|c|c|c|c|c|}
\hline Film material & $\begin{array}{l}\text { Device structure } \\
\text { (Bias condition) }\end{array}$ & Switching & $\begin{array}{c}\text { Forming process } \\
\text { Deg. fraction }\end{array}$ & Deg. fraction & $\begin{array}{l}\text { Reset process } \\
\text { E', E'” centers }\end{array}$ & Ov annihilated \\
\hline Silicon oxide & $\mathrm{A}(+4 \mathrm{~V} \rightarrow+4 \mathrm{~V})$ & Not work & $35.2 \%$ & $33.5 \%$ & 8 & 79 \\
\hline Silicon oxide & $\mathrm{B}(+3.5 \rightarrow+4 \mathrm{~V})$ & $U$ & $34.5 \%$ & $3.5 \%$ & 1353 & 3700 \\
\hline Silicon oxide & $\mathrm{A}(-4.5 \mathrm{~V} \rightarrow-4.5 \mathrm{~V})$ & Not work & $41.5 \%$ & $33.6 \%$ & 84 & 313 \\
\hline Silicon oxide & $\mathrm{C}(-3.65 \mathrm{~V} \rightarrow-4.3 \mathrm{~V})$ & $\mathrm{U}$ & $36.8 \%$ & $3.0 \%$ & 1623 & 3598 \\
\hline Silicon oxide & $\mathrm{A}(-4 \mathrm{~V} \rightarrow+4 \mathrm{~V})$ & Not work & $38.2 \%$ & $36.2 \%$ & 14 & 84 \\
\hline Silicon oxide & $\mathrm{B}(-4 \mathrm{~V} \rightarrow+4 \mathrm{~V})$ & B & $34.2 \%$ & $3.6 \%$ & 1345 & 3677 \\
\hline Silicon oxide & $\mathrm{C}(-3.65 \mathrm{~V} \rightarrow+3.7 \mathrm{~V})$ & B & $38.3 \%$ & $1.1 \%$ & 908 & 4098 \\
\hline Silicon oxide & $\mathrm{D}(-3.5 \mathrm{~V} \rightarrow+3.5 \mathrm{~V})$ & B & $34.9 \%$ & $0.65 \%$ & 824 & 4174 \\
\hline
\end{tabular}

\section{CONCLUSION}

This paper discussed the various impacts of hot-electron injection on the switching behavior of sputter-deposited silicon oxide films with the aid of Monte Carlo simulations. Switching behaviors of various stack structures were examined, and important parameters were summarized. Simulations yielded the following primary results. (a) Hot-electron injection is very useful in enabling the successful switching of sputter-deposited silicon oxide film. (b) Hot-electron injection promises a significant reduction in the switching energy of sputter-deposited silicon oxide films. (c) Two-layer stack of metals can significantly reduce the number of oxygen vacancies in the sputter-deposited silicon oxide film after the reset process. It is anticipated that the wide electron energy distribution, which is wider than the distribution assumed for device B, plays an important role. (d) In unipolar switching, the number of E' or E" centers of the sputter-deposited silicon oxide film is relatively large. This matches the prediction made in our previous papers. The results confirm that the Monte Carlo simulation model assumed in this paper is at least available for the analysis of resistive switching phenomena of silicon oxide films.

\section{REFERENCES}

[1] J. Yao, L. Zhong, D. Natelson, and J. M. Tour, "Etching-dependent reproducible memory switching in vertical $\mathrm{SiO}_{2}$ structures," Applied Physics Letters, vol. 93, pp. 253101-1-253101-3, 2008, doi: 10.1063/1.3045951.

[2] Y. Wang, et al., "Memory switching properties of e-beam evaporated $\mathrm{SiO}_{\mathrm{x}}$ on $\mathrm{n}^{++}$Si substrate," Applied Physics Letters, vol. 100, pp. 083502-1-083502-3, 2012, doi: 10.1063/1.3687724.

[3] A. Mehonic et al., "Resistance Switching in Silicon Sub-oxide Films," Journal of Applied Physics, vol. 111, pp. 074507-1-074507-9, 2012, doi: 10.1063/1.3701581. 
[4] Y. F. Chang et al., "Intrinsic SiOx-based unipolar resistance switching memory. II. Thermal effects on charge transport and characterization of multilevel programing," Journal of Applied Physics, vol. 116, pp. 043709-1-043709-12, 2014, doi: 10.1063/1.4891244.

[5] R. Yamaguchi, S. Sato, Y. Omura, and K. Nakamura, "Characterization and Modeling of Resistance-Switching Phenomena and Electronic Structure of Sputter-Deposition $\mathrm{SiO}_{2}$ Films," Tech. Dig., $11^{\text {th }}$ International Workshop on Low-Temperatureb Electronics (WOLTE-11), pp. 69-72, 2014, doi: 10.1109/WOLTE.2014.6881028.

[6] Y. F. Chang et al., "Intrinsic SiOx-based unipolar resistance switching memory. I. Oxide stoichiometry effects on reversible switching and program window optimization," Journal of Applied Physics, vol. 116, pp. 043708-1-043708-10, 2014, doi: 10.1063/1.4891242.

[7] H. Jiang et al., "Bias-polarity-dependent resistance switching in W/SiO2/Pt and W/SiO2/ Si/Pt structures," Scientic Report, vol. 6, no. 22216, 2016, doi: 10.1038/srep22216.

[8] C. He et al., "Multilevel Resistance Switching in Planar Graphene/SiO2 Nanogap Structures," ACS Nano, vol. 6, pp. 4214-4221, 2012, doi: 10.1021/nn300735s.

[9] Y.-E. Syu et al., "Asymmetric Carrier Conduction Mechanism by Tip Electric Field in WSiOx Resistance Switching Device," IEEE Electron Device Letters, vol. 33, pp. 342-344, 2012, doi: 10.1109/LED.2011.2182600.

[10] Y.-F. Chang et al., "Understanding the Resistance Switching Characteristics and Mechanism in Active SiOx-Based Resistance Switching Memory," Journal of Applied Physics, vol. 112, pp. 123702-1-123702-8, 2012, doi: 10.1063/1.4769218.

[11] B. W. Fowler et al., "Electroforming and resistance switching in silicon dioxide resistance memory devices," RSC Advances, vol. 5, pp. 21215-21236, 2015, doi: 10.1039/C4RA16078A.

[12] Y. Wang, X. Qian, K. Chen, Z. Fang, W. Li, and J. Xu, "Resistance Switching Mechanism in Silicon highly Rich SiOx $(x<0.75)$ Films Based on Silicon Dangling Bonds Percolation Model," Applied Physics Letters, vol. 102, pp. 042103-1-042103-5, 2013, doi: 10.1063/1.4776695.

[13] Y. Wang, K. Chen, X. Qian, Z. Fang, W. Li, and J. Xu, "The x Dependent Two Kinds of Resistance Switching Behaviors in $\mathrm{SiO}_{\mathrm{x}}$ Film with different $x$ Component," Applied Physics Letters, vol. 104, pp. 012112-1-012112-5, 2014, doi: 10.1063/1.4861592.

[14] A. Mehonic et al., "Structural changes and conductance thresholds in metal-free intrinsic $\mathrm{SiO}_{\mathrm{x}}$ resistance random access memory," Journal of Applied Physics, vol. 117, pp. 124505-1-125405-10, 2015, doi: 10.1063/1.4916259.

[15] A. Mehonic et al., "Nanoscale Transformations in Metastable Amorphous, Silicon-Rich Silica," Advanced Materials., vol. 28, pp. 7486-7493, 2016, doi: 10.1002/adma.201601208.

[16] D. Z. Gao, A.-M. El-Sayed, and A. L. Shluger, "A Mechanism for Frenkel defect creation in amorphous $\mathrm{SiO}_{2}$ facilitated by electron injection," Nanotechnology, vol. 27, pp. 505207-505210, 2016, doi: 10.1088/0957-4484/27/50/505207.

[17] A. Mehonic et al., "Silicon Oxide (SiOx): A Promising Material for Resistance Switching," Advanced Materials, vol. 2018, pp. 1801187-1-1801187-21, 2018, doi: 10.1002/adma.201801187.

[18] R. Yamaguchi, S. Sato, and Y. Omura, "Roles of chemical stoichiometry and hot electrons in realizing the stable resistance switching of sputter-deposited silicon oxide films," Jpn. J. Appl. Phys., vol. 56, pp. 041301-1-041301-6, 2017, doi: 10.7567/JJAP.56.041301.

[19] Y. Omura, T. Akano, and S. Sato, "Possible Models of Electron-Energy Transfer in Resistance Switching by Sputter-Deposited Silicon Oxide Films: Potential of Extremely Low-Energy Switching," ECS Journal of Solid State Science and Technology, vol. 7, pp. Q21-Q25, 2018, doi: 10.1149/2.0091803jss.

[20] Y. Omura, R. Yamaguchi, and S. Sato, "Study on the Impacts of Hole Injection and Inclusion of Sub-Oxide and Metallic Si Atoms on Repeatable Resistance Switching of Sputter-Deposited Silicon Oxide Films," IEEE Tranaction on. Devices and Materials Reliability, vol. 18, pp. 561-567, 2018, doi: 10.1109/TDMR.2017.2756260.

[21] Y. Omura, "Physics-based model for resistance transition of sputter-deposited silicon oxide films," Materials Today: Proceedings, vol. 20, Part 3, pp. 273-282, 2020, doi: 10.1016/j.matpr.2019.10.064.

[22] J. S. Lee, S. Lee, and T. W. Noh, "Resistive switching phenomena: A review of statistical physics approaches," Applied Physics Review, vol. 2, pp. 031303-1-031303-57, 2015, doi: 10.1063/1.4929512.

[23] Y.-F. Chang, B. Fowler, F. Zhou, Y.-C. Chen, and J. C. Lee, "Study of self-compliance behaviors and internal filament characteristics in intrinsic SiOx-based resistive switching memory," Applied Physics Letters, vol. 108, pp. 033504-1-033504-3, 2016, doi: 10.1063/1.4940203.

[24] F. Zhou et al., "A study of the interfacial resistive switching mechanism by proton exchange reactions on the $\mathrm{SiO}_{\mathrm{x}}$ layer," Physical Chemistry and Chemical Physics, vol. 18, pp. 700-703, 2016, doi: 10.1039/C5CP06507K.

[25] Y.-F. Chang, B. Fowler, Y.-C. Chen, and J. C. Lee, "Proton exchange reactions in SiOx-based resistive switching memory: Review and insights from impedance spectroscopy," Progress in Silid State Chemistry, vol. 44, pp. 75-85, 2016, doi: 10.1016/j.progsolidstchem.2016.07.001.

[26] Y.-F. Chang et al., "Beyond SiOx: an active electronics resurgence and biomimetic reactive oxygen species production and regulation from mitochondria," Journal of Materials Chemistry C, vol. 6, pp. 12788-12799, 2018, doi: $10.1039 / \mathrm{C} 8 \mathrm{TC} 04355 \mathrm{H}$.

[27] Y. Omura, "Possible Model of Degradation Mechanism that Increments the Gate Current (Non-Biased Interval) after Constant-Current Stress," Journal of Applied Physics, vol. 102, pp. 033710-1-033710-7, 2007, doi: $10.1063 / 1.2768007$.

[28] A. Mazady and M. Anwar, "Transient analysis of memristors," in Lester Eastman Conference on High Performance Devices (LEC) (Brown University, Singapore, Aug., 2012), doi: 10.1109/lec.2012.6410974.

[29] S. Larentis, F. Nardi, S. Balatti, D. C. Gilmer, and D. Ielmini, "Resistive switching by voltage-driven ion migration in bipolar RRAM-Part II: Modeling," IEEE Transaction on Electron Devices, vol. 59, pp. 2468-2475, 2012, doi: 10.1109/TED.2012.2202320. 
[30] S. Lavizzari, D. Ielmini, and A. L. Lacaita, "Transient simulation of delay and switching effects in phase-change memories," IEEE Transaction on Electron Devices, vol. 57, pp. 3257-3264, 2010, doi: 10.1109/TED.2010.2078822.

[31] X. Yang, "Demonstration of Ultra-Fast Switching in Nanometallic Resistive Switching Memory Devices," Journal of Nanoscience (Hindawi), vol. 2016, 2016, doi: 10.1155/2016/8132701.

[32] S. Yu, X. Guan, and H.-S. P. Wong, "On the stochastic nature of resistive switching in metal oxide RRAM: Physical modeling, Monte Carlo simulation, and experimental characterization," 2011 IEEE International Electron Device Meeting (Washington, D. C., 2011), p. 413-416, doi: 10.1109/IEDM.2011.6131572.

[33] W. H. Baur, "Variation of Mean Si-O Bond Lengths in Silicon-Oxygen Tetrahedra," Acta Crystallographica B, vol. 34, pp. 1751-1756, 1978, doi: 10.1107/S0567740878006640.

[34] W. M. H. Sachtler, G. J. H. Dorgelo, and A. A. Holscher, "The work function of gold," Surface Science, vol. 5, pp. 211-219, 1966, doi: 10.1016/0039-6028(66)90083-5.

[35] H. Ma et al., "Self-Rectifying Resistive Switching Memory with Ultralow Switching Current in Pt/Ta2O5/HfO2-x /Hf Stack," Nanoscale Research Letters, vol. 12, pp. 118-1-118-6, 2017, doi: 10.1186/s11671-017-1905-3.

[36] J. W. McPherson and C. Mogul, "Underling Physics of the Thermochemical E Model in Describing Low-Field Time-Dependent Dielectric Breakdown in SiO2 Thin Films," Journal of Applied Physics, vol. 84, pp. 1513-1523, 1998, doi: 10.1063/1.368217.

[37] A. V. Kimmel, P. V. Sushko, A. L. Shluger, and G. Bersuker, "Positive and negative oxygen vacancies in amorphous silica," ECS Trans, vol. 19, no. 2, pp. 3-17, 2009, doi: 10.1149/1.3122083.

[38] A.-M. El-Sayed, Y. Wimmer, W. Goes, T. Grasser, V. V. Afanas'ev, and A. L. Shluger, "Theoretical models of hydrogen-induced defects in amorphous silicon dioxide," Physical Reviews B, vol. 92, pp. 014107-1-014107-11, 2015, doi: 10.1103/PhysRevB.92.014107.

[39] K. Komiya and Y. Omura, "Spectroscopic characterization of stress-induced leakage current in sub-5-nm-thick silicon oxide film," Journal of Applied Physics, vol. 92, pp. 2593-2601, 2002, doi: 10.1063/1.1499978.

[40] "International Technology Roadmap for Semiconductors", semicond. Industry Association, 2003, https://www.semiconductors.org/

[41] J. H. Stathis, "Percolation models for gate oxide breakdown," Journal of Applied Physics, vol. 86, pp. 5757-5766, 1999, doi: 10.1063/1.371590.

[42] D. B. Strukov, G. S. Snider, D. R. Stewart, and R. S. Williams,"The Missing Memristor Found," Nature, vol. 453, pp. 80-83, 2008, doi: 10.1038/nature06932.

\section{BIOGRAPHY OF AUTHOR}

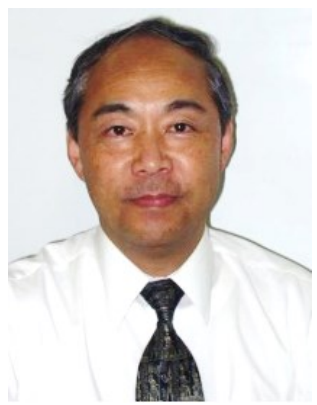

Yasuhisa Omura was with NTT Telecommunication Laboratoties in Musashino and Atsugi from 1975 to 1997 . He contributed to Si device technology development, advancing siliconon-insulator (SOI) technology, and device physics. He moved his position to Kansai University in Osaka as a professor in 1997. He served the Director of High-Technology Center in Kansai University for 5 years. He retired from Kansai University in 2019, and is now with Academic Collaboration Associates, Japan. He has published 170 journal papers, 200 international conference papers, and contributed to several book editions. He also published, as a primary editor, two books from IEEE/Wiley in 2013 and 2016. 